\title{
Schrödinger Equation and (Future) Quantum Physics
}

\author{
Miloš V. Lokajíček, Vojtěch Kundrát and \\ Jiří Procházka \\ Additional information is available at the end of the chapter
}

http://dx.doi.org/10.5772/53844

\section{Introduction}

The Schrödinger equation based on the Hamiltonian taken from the classical physics provides the solutions that may be correlated to the solutions of Hamilton equations or to their superpositions, if the additional assumptions introduced by Bohr have not been added; i.e., simple Hilbert space spanned on one set of Hamiltonian eigenfunctions and each vector representing pure state. It may describe, therefore, physical processes at microscopic as well as macroscopic levels; only the set of allowed states being partially limited. However, the given equation represents approximative phenomenological theory that is not able to explain the emergence of quantum states on the basis of Coulomb potential only; e.g., in the case of hydrogen atom such a state may arise evidently only if a repulsive short-ranged force (or some contact interaction) exists between electron and proton at low kinetic energy values. The dimensions and other properties of proton should play then important role in such a case. These characteristics may be derived from different experiments studying collisions between corresponding objects at different energies. They should be taken into account when the quantum physics is to pass from hitherto mere phenomenological description of physical evolution to causal realistic interpretation as it was common in classical physics. It concerns mainly the spontaneous transitions between different quantum states in atoms.

However, let us start with short summary of main points concerning the evolution of quantum mechanics theory during the 20th century. The Copenhagen quantum mechanics (based fundamentally on the Schrödinger equation [1] and proposed by Bohr [2] in 1927) has been taken as the only theory of microscopic physical processes till the end of the 20th century, even if it has involved a series of paradoxes corresponding in principle to internal contradictions contained in corresponding mathematical model. Already in 1933 Pauli [3] showed that the corresponding Hamiltonian was to exhibit continuous energy spectrum in the whole interval $E \in(-\infty,+\infty)$, which contradicted the existence of quantized energy 
states in closed systems. However, it has been possible to say that this fact did not raised any greater interest at that time.

More attention was devoted to the criticism of Einstein [4] in 1935, who showed with the help of a Gedankenexperiment that the given quantum theory required the existence of immediate interaction (or rather linkage) between two very distant matter objects, which was denoted by him as unacceptable on the basis of standard ontological experience. The given critique was refused by Bohr [5] having argued that this distant immediate interaction might exist between microscopic objects (at the difference to macroscopic phenomena). The world scientific community accepted fully Bohr's standpoint that was supported partially by the earlier argument of von Neumann [6] who refused the existence of any local (hidden) parameters in Copenhagen theory; the problem having been discussed several years ago. The argument of Grete Herrmann that the given conclusion of von Neumann was based on circular proof [7] was not taken into account, having remained practically unknown at that time.

A partial change occurred in 1952 when Bohm [8] showed that an additional (hidden) parameter existed already in the simplest solutions of Schrödinger equation. Two alternatives (Copenhagen mechanics and hidden-variable theory) were then considered in the microscopic region. The decision between them was expected to be done on the basis of experimental results when Bell [9] derived his inequalities in 1964 which were assumed to hold in the hidden-variable theory and not in the Copenhagen alternative. The corresponding experiments based in principle on the original proposal of Einstein were finished in 1982 and the inequalities of Bell were provably violated [10]. The Copenhagen quantum mechanics was then taken as the only physical theory valid for microscopic physical processes.

Only in the end of the last century it was shown for the first time that the given conclusion was based on the mistaking assumption and that the given inequalities did not hold in any quantum alternative based on Schrödinger equation [11]. In 2004 it was then shown by Rosinger [12] that Bell's inequalities contradicted also the inequalities derived by Boole (in 1854) for any probabilistic system $[13,14]$ :

$$
\begin{gathered}
\max \left\{p_{1}, p_{2}, \ldots ., p_{n}\right\} \leq P\left(A_{1} \cup A_{2} \cup \ldots \cup A_{n}\right) \leq \\
\min \left\{1, p_{1}+p_{2}+\ldots . .+p_{n}\right\} \\
\max \left\{0, p_{1}+p_{2}+\ldots .+p_{n}-n+1\right\} \leq \\
P\left(A_{1} \cap A_{2} \cap \ldots . \cap A_{n}\right) \leq \min \left\{1, p_{1}, p_{2}, \ldots . ., p_{n}\right\}
\end{gathered}
$$

where the first relation concerns the probabilities $p_{j}$ of possible alternative phenomena $A_{j}$ and the other one concerns those of contemporary phenomena.

More detailed analysis of the Bell inequalities problem may be found in [15]. It has been possible to conclude that these inequalities have been valid only in the classical physics, not in any kind of quantum theory. They have been mistakenly applied to experiment where photon polarizations have been measured while this possibility has been excluded by the assumption on the basis of which they were derived.

The consequences following from this fact have been summarized in [16]; more complete summary of all problems of contemporary quantum mechanics has been then presented in 
[17]. It has followed from these results that the Schrödinger equation itself may be denoted as common theory of all physical reality when the Hilbert space formed by its solutions has been adapted to given physical system (i.e., correspondingly extended) in contradiction to limiting and deforming conditions imposed by Bohr.

The given conclusion has followed practically from the fact that the Schrödinger equation may be derived for the set of statistical combinations of Hamilton equation solutions when the given set has been limited by a suitable condition (e.g., by Boltzmann statistics); see $[18,19]$. It means that any Schrödinger function $\psi(x, t)$ may represent always a classical state or a statistical combination of such states; see also [20] (or already quoted [17]).

However, the Schrödinger equation (including Coulomb potential only) represents approximative phenomenological theory only. It is not able to explain any emergence of quantum energy states. In such a case some additional properties of individual objects forming a quantized system must be taken into account. E.g., in the case of hydrogen atom any quantum state could not come into being only on the basis of Coulomb force if some further repulsive or contact interaction did not exist between electron and proton that would depend necessarily on dimensions and structures of main constituents. The study of corresponding structures of individual objects should represent, therefore, inseparable part of contemporary quantum physics.

These structures may be derived mainly from the data gained in experiments concerning the collisions between corresponding particles. However, it is not sufficient to look for a phenomenological description of some measured values. More detailed collision models must be made use of in such a case. They must be able to study the dependence on impact parameter value; e.g., at least the so called eikonal model should be made use of. Some results obtained on the given realistic basis will be introduced in the following; it will be discussed how they may be made use of in solving the problems of quantum physics in the next future.

In Sec. 2 we shall start with discussing the significance of the Schrödinger equation, based on the fact that it may be derived in principle from Hamilton equations. The necessity of repulsive force in the emergence of quantum states in closed systems will be then discussed in Sec. 3. The eikonal model of elastic collisions between hadrons and some results obtained for elastic proton-proton collisions in the impact parameter space will be presented in Sec. 4 . The problem of validity of optical theorem on which practically all contemporary elastic collision results have been based will be discussed in Sec. 5. In Sec. 6 new probabilistic ontological model enabling to study the existence of internal proton structures will be applied to experimental data and new results will be presented. In Sec. 7 some metaphysical consequences will be considered. Several open questions will be then mentioned in Sec. 8 .

\section{Schrödinger equation and Hilbert space structure}

The evolution of a physical system consisting of different matter objects may be described with the help of Hamilton equations

$$
\dot{q}_{j}=\frac{\partial H}{\partial p_{j}}, \quad \dot{p}_{j}=-\frac{\partial H}{\partial q_{j}}
$$


where $q_{j}$ and $p_{j}$ are space coordinates and momenta of all individual objects; the corresponding Hamiltonian given by

$$
H=\frac{p_{j}^{2}}{2 m}+V\left(q_{j}\right)
$$

represents the total energy of the given system.

Schrödinger equation may be then written as

$$
i \hbar \frac{\partial \psi(x, t)}{\partial t}=H \psi(x, t)
$$

where $x$ represents the set of coordinates and $q_{j}$ and $p_{j}$ in the Hamiltonian are substituted by operators $q_{o p}^{j}=x_{j}$ and $p_{o p}^{j}=-i \hbar \frac{\partial}{\partial x_{j}}$. Time-dependent physical quantities are then defined as expectation values of corresponding operators

$$
A(t)=\int \psi^{*}(x, t) A_{o p} \psi(x, t) d x
$$

where $A_{o p}$ is represented by corresponding combination of operators $q_{o p}^{j}$ and $p_{o p}^{j}$.

It has been introduced in the preceding section that any solution $\psi(x, t)$ of Schrödinger equation may be identified with a solution of Hamilton equations or with a superposition of these classical solutions. Any function $\psi(x, t)$ at a given $t$ may be then represented by a vector in the correspondingly constructed Hilbert space. This Hilbert space must consist of several mutually orthogonal Hilbert subspaces, each of them being spanned on the set of Hamiltonian eigenfunctions:

$$
H \psi_{E}\left(x_{j}\right)=E \psi_{E}\left(x_{j}\right) .
$$

Two identical $\psi(x, t)$ functions exhibiting opposite time derivatives must belong always to different subspaces (i.e., incoming and outgoing states of evolving physical system must be taken as fundamentally different). The arrangements of total Hilbert spaces differ somewhat for continuous and discrete parts of Hamiltonian energy spectrum; corresponding details being found in [21, 22].

In the system of two free particles in their center-of-mass system, e.g., the Hilbert space must consist of two mutually orthogonal Hilbert subspaces (see also [23, 24]) being formed by incoming and outgoing states:

$$
\mathcal{H} \equiv\left\{\Delta^{-} \oplus \Delta^{+}\right\} ;
$$

the given subspaces being mutually related with the help of evolution operator

$$
U(t)=e^{-i H t}
$$


The individual vectors of evolution trajectory correspond then to the different expectation values of time operator $T$ fulfilling the condition

$$
i[H, T]=1
$$

where the state corresponding to zero value of $T$ may be arbitrarily chosen; being usually attributed to the state when mutual particle distance (impact parameter) is minimal. Eq. (8) defines the time unit for a corresponding physical system; or for any subsystem that evolves independently of the whole greater system.

Any time evolution trajectory may be characterized by the Hamiltonian expectation value E that is conserved during the whole evolution. It is, of course, also the expectation value of the angular momentum operator

$$
M^{2}=M_{i} M_{i}, \quad M_{i}=\varepsilon_{i j k} q_{o p}^{j} p_{o p}^{k}
$$

commuting with the Hamiltonian

$$
\left[M^{2}, H\right]=0
$$

that is conserved. In principle it holds also $\left[M_{i}, H\right]=0$ and $\left[M_{i}, M^{2}\right]=0$; thus one of components $M_{i}$ should be also conserved. However, its value depends on the orientation of the coordinate system. It is always possible to choose it so that the expectation value of given $M_{i}$ is maximal. It means that any evolution trajectory of a given physical system is defined in addition to energy $E$ also by the value of $M^{2}$; and in principle also by the sign of corresponding $M_{i}$.

As to the closed physical systems they are standardly taken as corresponding to the discrete part of Hamiltonian spectrum. In such a case the Hilbert space should be at least doubled in comparison to Eq. (6) as two different kinds of incoming and outgoing states exist; for more details see [21, 22]. Some other problems concerning closed physical systems (e.g., their emergence) will be discussed in the next section.

Let us return, however, to the problem of free two-particle system. It is necessary to mention one additional possibility when two incoming particles having corresponding kinetic energy may form an unstable object that decays after a short time. In such a case the Hilbert space may be further extended:

$$
\mathcal{H} \equiv\left\{\Delta^{-} \oplus \Theta \oplus \Delta^{+}\right\}
$$

where $\Theta$ may represent the object (or physical system) arisen by merging of two incoming particles (belonging to $\Delta^{-}$subspace). This new object (or system) may be stable or (according to available free energy) unstable decaying into a state lying in $\Delta^{+}$or creating a further kind of physical systems or objects. The terms representing corresponding transition probabilities between different Hilbert subspaces must be added to earlier (classical) potential between two particles.

In this case it is, of course, necessary to respect always that the evolution goes in irreversible way. Evolution of the pair of colliding particles is described in the beginning in $\Delta^{-}$, particles 
going nearer one to the other (incoming). In dependence on impact parameter value they are scattered by mutual potential and continue in $\Delta^{+}$as outgoing pair. However, in the case of small impact parameter values (at corresponding energy values) they may form also one common object being represented by a vector in Hilbert subspace $\Theta$. The corresponding evolution may be described, of course, at the present on phenomenological level only as we do not know actual internal dynamics of arising object.

The object represented by the subspace $\Theta$ may be stable (e.g., creation of atom from nucleus and electron) or unstable (decaying in the same particle pair or in another one). At much higher energy values many other particles may be, of course, formed. In such a case the subspace $\Theta$ is to be substituted by a more complex system of products (or sums) of corresponding subspaces describing further evolution of separated physical systems.

While the simple subspaces (in which the interacting particles remain stable) may be in principle described with the help of corresponding Schrödinger equations the other processes require to be characterized by additional probabilities between concrete states in individual subspaces. There is not any interference between amplitudes from different orthogonal subspaces.

The individual (stable as well as unstable) objects represent closed physical systems that are characterized by some quantum physical values. Each object has some internal dynamics (eventually, exists in some different internal states - stable or unstable). And just these questions represent evidently one of the main problems of the future quantum physics.

\section{Closed systems and quantum states}

The idea of quantum states has been based on experimental data concerning the measured light spectra emitted by excited atoms, as it was formulated in two phenomenological postulates of N. Bohr [25]. These spectra have been correlated to transitions between different quantum energy levels. The existence of quantum states have been then derived with the help of Schrödinger equation containing Coulomb potential.

However, it is necessary to call attention to the fact that the Schrödinger equation provides an approximative phenomenological description of quantum phenomena only. It predicts and admits the existence of quantum states but it cannot explain at all how such a quantum state may arise when two corresponding objects (forming then the closed system) are mutually attracted and go always nearer one to the other. It is evident that the emergence of quantum state is not possible without the conjoint existence of short-ranged repulsive (or contact) force acting between these objects, too. One should expect that both the corresponding potentials (forces) are to be responsible for the final effect.

In the standard approach the corresponding quantum states are represented by special trajectories of electrons orbiting around a nucleus. It means that the quantum energy value should be correlated to a special value of angular momentum. It should be correlated also to dimensions of the physical system in the given state (and also to the dimensions of individual objects), which does not seem to have been analyzed sufficiently until now. It relates probably to the emergence of quantum states, which represents open question, too.

Let us start with this last problem in the case of hydrogen atom consisting of one electron and one proton. A stable hydrogen atom should arise always when a slowly moving electron 
appears in the neighborhood of a proton. The electron is attracted to a proton and it is evident that the given atom might be hardly formed if a repulsive (short-ranged) force between electron and proton did not exist. Some contact linkage of these two objects (e.g., some adhesive force between them) should be also taken into consideration.

At the present any force is being interpreted as the result of some potential. In such a case it might be expected that corresponding quantum states correspond to the distance when both these potentials compensate:

$$
V(x)=V_{\text {Coul }}(x)+V_{\text {rep }}(x) \cong 0 \text {. }
$$

The corresponding quantum states should then depend on the shapes of these two potentials. And we should ask how they might be influenced, e.g., by the proton or by its internal states that might be changeable as one must expect for the proton to exhibit some internal dynamics. The other question concerns then the problem how the energy of emitted photon is determined to correspond to the difference of corresponding quantum energy values and further which atom constituent emits the given photon.

It seems, therefore, that to expect for the quantum states to be mediated by some potential acting at the distance may be hardly convenient. The other possibility, i.e., the existence of weak adhesive force between electron and proton, might be more acceptable. In such a case the properties of hydrogen atom should follow from the properties of proton and its internal dynamics. The changes in the given adhesive linkage might be then responsible also for the energy of emitted photons.

In both the cases it is, of course, necessary to expect that the quantum states of hydrogen atom are to be fundamentally dependent on the dimensions and structure of proton. One must, therefore, assemble and analyze all corresponding characteristics that may be derived from available experimental data.

The characteristics of proton may be studied mainly with the help of experiments concerning the collisions between electrons and protons or between two protons. Unfortunately, in the corresponding analyzes only some mathematical models are usually used that describe some average phenomenological characteristics of given particles, which can hardly contribute to understanding the discussed problems of quantum physics. The models respecting the size and structures of individual particles must be made use of.

In the last time we have studied the elastic proton-proton collisions at higher values of collision energy using the eikonal model where the probability of different processes in the dependence on impact parameter values may be derived [26]; some conclusions differing from earlier ones having been obtained. First of all it has been demonstrated that there is not any reason for arguing that the elastic processes should be central, i.e., existing at very small (even zero) values of impact parameter. When any limiting condition (facilitating the calculations) has not been imposed the elastic collisions may be interpreted as peripheral in full agreement with ontological interpretation of microscopic objects; see more details in the next section. 


\section{Proton-proton collisions in impact parameter space}

In this section we shall present some results of elastic proton-proton collisions in the impact parameter space obtained earlier. The corresponding experimental data are represented by elastic differential cross section that is in the given case given by two different mutual interactions: Coulomb and strong ones. If the influence of proton spins is neglected one measures and establishes the dependence $\frac{\mathrm{d} \sigma^{C+N}(s, t)}{\mathrm{d} t}$ where $s$ is the square of center-of-mass energy and $t$ is the square of center-of-mass four-momentum transfer (it is zero or negative and it is a function of scattering angle).

It is not possible to measure the influence of individual interactions separately. In the standard theoretical framework any collision process is regarded as fully described provided its complex scattering amplitude is given. The measured differential cross section is written as

$$
\frac{\mathrm{d} \sigma^{C+N}(s, t)}{\mathrm{d} t}=\frac{\pi}{s p^{2}}\left|F^{C+N}(s, t)\right|^{2}
$$

where $p$ is the three-momentum of incident nucleon in the center-of-mass system $\left(s=4\left(p^{2}+\right.\right.$ $\left.m^{2}\right) ; m$ being mass of a proton); natural units have been used: $\hbar=c=1$. In the case of only hadronic (resp. Coulomb) elastic scattering it is, therefore, necessary to know amplitude $F^{N}(s, t)$ (resp. $F^{C}(s, t)$ ). And one must ask how to express complete amplitude $F^{C+N}(s, t)$ with the help of individual amplitudes $F^{N}(s, t)$ and $F^{C}(s, t)$.

Formulas still standardly used for complete elastic scattering amplitude $F^{C+N}(s, t)$ were derived under several very limiting assumptions. One of such commonly used formulas has been the simplified formula of West and Yennie (WY) published in 1968 [27] which was derived only for very low values of $|t|$ under assumption that the modulus of $F^{N}(s, t)$ was purely exponential in $t$ and the phase of $F^{N}(s, t)$ was $t$-independent; both these assumptions were supposed to be valid in the whole region of kinematically allowed values of $t$ at that time. Detailed discussion concerning both theoretical and experimental problems following from drastic limitation involved in this formula may be found, e.g., in [28].

The question has been raised whether it is possible to derive more general formula without any a priory limitation on $F^{C+N}(s, t)$, which could be used for more relevant analysis of experimental data. It has been possible to remove the most of earlier limitations if eikonal model has been applied to. According to [26] (in 1994) it has been possible to derive on the basis of the eikonal model more general formula for the complete elastic amplitude for any $s$ and $t$ with the accuracy up to terms linear in $\alpha$ and without any a priory restriction on hadronic amplitude $F^{N}(s, t)$.

If this hadronic amplitude $F^{N}(s, t)$ is given then some physically significant quantities may be calculated from it. For example, one may calculate distribution functions of total, elastic and inelastic hadronic collisions in the impact parameter space and if they are determined then one may evaluate mean-squares of impact parameter for corresponding processes. The mean-square of impact parameter in the case of elastic processes may be calculated from corresponding elastic distribution function $D_{e l}(s, b)$ as 


$$
\left\langle b^{2}(s)\right\rangle_{e l}=\frac{\int_{0}^{\infty} b \mathrm{~d} b b^{2} D_{e l}(s, b)}{\int_{0}^{\infty} b \mathrm{~d} b D_{e l}(s, b)} .
$$

and similarly we may define also $\left\langle b^{2}(s)\right\rangle_{\text {tot }}$ and $\left\langle b^{2}(s)\right\rangle_{\text {inel }}$ in the case of total and inelastic hadronic collisions. However, in eikonal model the mean-squares of all these processes may be derived directly from the $t$-dependent elastic hadronic amplitude $F^{N}(s, t)$ without trying to establish the whole distribution functions, which is mathematically much more complicated; detailed discussion how to actually determine all distribution functions on the basis of corresponding experimental data can be found in [29]. For a given hadronic amplitude $F^{N}(s, t)$ one may thus calculate relatively easily root-mean-squares $\sqrt{\left\langle b^{2}(s)\right\rangle_{e l}}$ and $\sqrt{\left\langle b^{2}(s)\right\rangle_{\text {inel }}}$ and compare both the values. If the value $\sqrt{\left\langle b^{2}(s)\right\rangle_{e l}}$ is lesser than $\sqrt{\left\langle b^{2}(s)\right\rangle_{\text {inel }}}$ then it would mean that elastic hadronic processes should be realized in average at lower impact parameter values than inelastic processes; the protons should be rather "transparent" which might be hardly acceptable from the ontological point of view. We may denote this situation as "central" behavior of elastic scattering. If the value $\sqrt{\left\langle b^{2}(s)\right\rangle_{e l}}$ is greater than $\sqrt{\left\langle b^{2}(s)\right\rangle_{\text {inel }}}$ then we denote situation as "peripheral" behavior of elastic scattering; the situation being in agreement with usual interpretation of two matter object collisions.

As already mentioned the corresponding proton-proton collision data have been interpreted with the help of rather simplified phenomenological mathematical models in the past. Some conclusions have been rather surprising. Especially, when it has been concluded that a rather great ratio of elastic processes has corresponded to purely central collisions (i.e., protons could scatter elastically even at impact parameter $b=0$ ). This kind of "transparency" of protons has been denoted already in 1979 in [30] as a "puzzle". In 1981 it has been then shown that the corresponding result has depended mainly on the $t$-dependence of the phase of elastic hadronic scattering amplitude, see [31]. The mentioned central behavior has been derived when the $t$-dependence of the phase has been strongly limited. And it has been shown that if the modulus is purely exponential and hadronic phase is practically $t$-independent in the whole region of kinematically allowed values of $t$ (at arbitrary collision energy $\sqrt{s}$ ) one obtains necessarily the mentioned central behavior of elastic hadronic collisions in the impact parameter space. These two (over)simplified assumptions have been commonly used in many analysis of corresponding experimental data (they are included also in the simplified WY formula).

Consequently, it has been very interesting to put the opposite question: How to modify the given mathematical model to obtain collision processes corresponding to usual ideas. The experimentally established elastic proton-proton differential cross section at energy of $\sqrt{s}=53 \mathrm{GeV}$ has been analyzed in the whole measured $t$-range with the help of more general eikonal formula used for complete scattering amplitude in [26]. It was possible to obtain acceptable fits for different $t$-dependencies of the phase (according to chosen parametrization). Two quite different dependencies of hadronic phase have been then shown in the quoted paper. The first phase was the so-called "standard" phase (used, e.g., in [32] for interpretation of experimental data) and the second phase corresponded to natural peripheral elastic collisions. Both the hadronic phases are plotted in Fig. 1. The root-mean-squares 


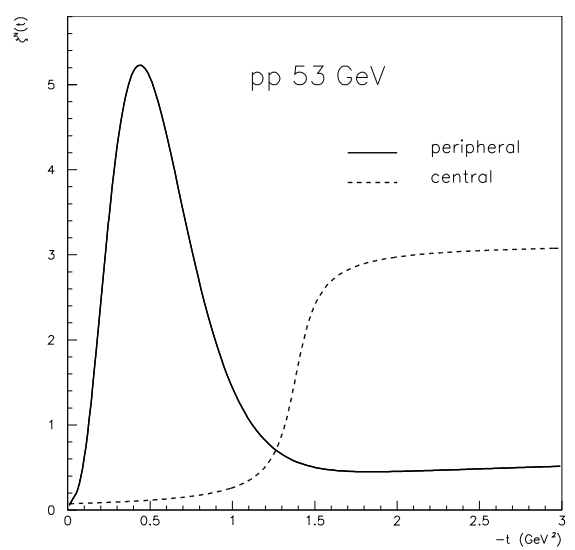

Figure 1. Two different hadronic phases $\zeta^{N}(s, t)$ defined by $F^{N}(s, t)=i\left|F^{N}(s, t)\right| e^{-i \zeta^{N}(s, t)}$ and fitted to corresponding experimental data at energy of $\sqrt{s}=53 \mathrm{GeV}$. Dashed line - standard hadronic phase admitting central elastic hadronic collisions; full line - peripheral phase.

corresponding to the two hadronic phases are then given in Table 1. The case with the standard hadronic phase leads to central elastic hadronic collisions. Similar result as in the case of proton-proton collisions was obtained also in the case of elastic antiproton-proton scattering at energy of $541 \mathrm{GeV}$ (see [26, 29]). The phase which leads to peripheral behavior of hadronic proton-proton collisions has very similar $t$-dependence as that obtained earlier at lower collision energy of $\sqrt{s}=23 \mathrm{GeV}$ in the already quoted older paper [31].

\begin{tabular}{lccccc}
\hline Data & Hadronic phase & Profile & $\sqrt{\left\langle b^{2}(s)\right\rangle_{\text {tot }}}$ & $\sqrt{\left\langle b^{2}(s)\right\rangle_{\text {el }}}$ & $\sqrt{\left\langle b^{2}(s)\right\rangle_{\text {inel }}}$ \\
\hline$p p 53 \mathrm{GeV}$ & peripheral & peripheral & 1.028 & 1.803 & 0.772 \\
$p p 53 \mathrm{GeV}$ & standard & central & 1.028 & 0.679 & 1.087 \\
$\bar{p} p 541 \mathrm{GeV}$ & peripheral & peripheral & 1.140 & 2.205 & 0.609 \\
$\bar{p} p 541 \mathrm{GeV}$ & standard & central & 1.140 & 0.756 & 1.220 \\
\hline
\end{tabular}

Table 1. Values of root-mean-squares (in femtometers) obtained from analysis of experimental data with standard and peripheral hadronic phase.

\begin{tabular}{lccc}
\hline Model & $\sqrt{\left\langle b^{2}(s)\right\rangle_{\text {tot }}}$ & $\sqrt{\left\langle b^{2}(s)\right\rangle_{e l}}$ & $\sqrt{\left\langle b^{2}(s)\right\rangle_{\text {inel }}}$ \\
\hline Bourelly et al. & 1.249 & 0.876 & 1.399 \\
Petrov et al. (2P) & 1.227 & 0.875 & 1.324 \\
Petrov et al. (3P) & 1.263 & 0.901 & 1.375 \\
Block et al. & 1.223 & 0.883 & 1.336 \\
Islam et al. & 1.552 & 1.048 & 1.659 \\
\hline
\end{tabular}

Table 2. Values of root-mean-squares (in femtometers) predicted by different models of proton-proton collisions at collision

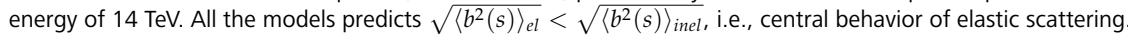

The elastic and inelastic root-mean-squares have been calculated in [28] also for several relatively new phenomenological models proposed for elastic hadronic proton-proton scattering at energy of $14 \mathrm{TeV}$ (planned ultimate LHC collision energy). In all these cases it has been found that $\left\langle b^{2}(s)\right\rangle_{e l}<\left\langle b^{2}(s)\right\rangle_{\text {inel }}$, i.e., all these models correspond to central behavior of proton collisions, as shown in Table 2 . 
One of the results obtained in [26] was that the modulus of hadronic amplitude $\left|F^{N}(s, t)\right|$ was practically uniquely determined from corresponding measured elastic differential cross section while the hadronic phase remained quite undetermined, being only slightly limited due to Coulomb interaction. It should be determined on the basis of other physical requirements, not on the basis of some arbitrary assumptions. The results gained in elastic proton-proton collisions might be very helpful for nuclear and particle physics in general. However, practically in all current descriptions of proton-proton elastic scattering the validity of optical theorem which relates the imaginary part of hadronic amplitude to total hadronic cross section, has been taken as a basic assumption. The given problem will be discussed in the next section, as the given validity does not seem to be sufficiently reasoned.

As to the other problem mentioned in the end of preceding section (the use of mathematical models representing only average structure of colliding objects) it has been at least partially removed when the more general eikonal model has been applied to and the results in dependence on impact parameter values have been established. However, the main character of some average phenomenological characteristics obtained from hitherto phenomenological models remains, which can hardly contribute to understanding the results of contemporary quantum physics. A new more detailed model of proton-proton elastic collisions with realistic behavior of the collisions in the impact parameter space and respecting the sizes and structures of individual colliding particles will be formulated and applied in preliminary form to experimental data in Sec. 6.

\section{Optical theorem and its validity}

Practically all hitherto mathematical models of proton collision processes (including the eikonal model described in Sec. 4) have been based on the assumption of optical theorem validity. This theorem has been taken from optical approaches where the total cross section was correlated to the imaginary part of complex index of refraction (see, e.g., the description of the given problem in [35]) and the behavior of light was studied on wave basis. The optical theorem has been applied then also to elastic particle collisions when the wave behavior has been attributed to all physical objects.

The approaches of deriving the validity of optical theorem in particle collisions have been summarized recently, e.g., in [36]. The goal of these approaches has consisted in deducing that it has held for the total cross section

$$
\sigma^{t o t}(s)=\sigma^{e l}(s)+\sigma^{i n e l}(s)=\frac{4 \pi}{p \sqrt{s}} \Im F(s, t=0)
$$

where the function $F(t)$ defines the elastic differential cross section

$$
\frac{d \sigma}{d t}=\frac{\pi}{s p^{2}}|F(s, t)|^{2}
$$

It holds then also

$$
F(s, t)=2 \pi \sqrt{s} \int_{0}^{\infty} b d b J_{0}(b \sqrt{-t}) \Gamma(b) .
$$


where $\Gamma(b)$ is the profile function in impact parameter space characterizing the probability of elastic processes in dependence on impact parameter values and on their initial distribution.

Until now there is not any reliable theory of elastic scattering and only different phenomenological models are practically available. The complex function

$$
F(s, t)=i|F(s, t)| e^{-i \zeta(s, t)}
$$

is being, therefore, derived usually from corresponding experimental data. However, from them only the modulus may be established while the phase $\zeta(t)$ remains quite undetermined. It is to be predicted on the basis of additional assumptions that may influence strongly the conclusions concerning the value of total cross section. Also the optical theorem has been practically always involved even if it has been derived on the basis of other additional assumptions that have not corresponded to actual characteristics of strong interactions as it was mentioned already earlier in [37].

In all attempts to derive the validity of optical theorem in particle collisions the standard approaches based on the Copenhagen quantum mechanics have been made use of; especially, the summation of amplitudes used commonly in S-matrix theory has been applied to. The states belonging to two mutually orthogonal Hilbert subspaces $\Delta^{-}$and $\Delta^{+}$(see Sec. (2)), i.e., the states at time $\tau=-\infty$ and at time $\tau=+\infty$, were taken as lying in one common Hilbert space and corresponding amplitudes were superposed, which cannot be done if the ontological approach is to be respected (i.e., time evolution described correctly with the help of time-dependent Schröedinger equation). It may be then denoted as surprising that the optical theorem has been applied to strong interaction only and never considered in the case of Coulomb interaction, even if the given derivation approach does not relate to a kind of interaction.

The final effect in collision processes of two protons is to depend on the conditions in individual events characterized mainly by corresponding impact parameter value. However, only the Coulomb elastic scattering comes into account for greater values of $b$ where short-ranged (or contact) strong interaction cannot exist while for lower values of $b$ elastic interaction (Coulomb or hadronic) may exist together with inelastic one. Consequently, as to the strong interaction the particles may continue in original motion practically without any disturbance; it means that such states represent a special set of states in the subspace $\Delta^{+}$which cannot be added if the total cross section corresponding to strong interaction is considered. Only the states scattered elastically by strong interaction may be involved.

It has been mentioned that for a given collision energy $\sqrt{s}$ only the modulus of hadronic amplitude $F(s, t)$ may be established from experimental data and the hadronic phase $\zeta(s, t)$ may be practically arbitrary. It means that very different characteristics may be attributed to elastic particle collisions; see the problem of centrality and peripherality of elastic hadronic processes discussed in preceding section. The choice of phase $t$-dependence has been then fundamentally influenced also by the application of the optical theorem, mainly in the region of very low values of $|t|$ where the measurement cannot be done. Even the separation of Coulomb and strong interactions might be fundamentally influenced when the parametrization of the strong part of scattering amplitude has allowed only the differential cross section decreasing monotony from the maximum value at $t=0$. In the case of 
short-ranged (contact) strong interactions it is necessary to admit that the maximum value may lie at non-zero value of $t$ as it will be demonstrated in the next section.

\section{Probabilistic ontological model of elastic proton-proton collisions}

It follows from the preceding that the Schrödinger equation leads to the same results as classical physics. However, in the classical physics based fully on ontological approach the individual matter objects represent always only the source of corresponding potentials mediating corresponding interactions. It may be applied fully to electromagnetic or gravitational forces. The strong (and also weak) interactions differ, however, rather strongly from these interactions. The short-ranged effect of the latter ones indicates that these forces should be denoted rather as contact ones; being zero at any greater distance. It means that in elastic proton-proton collisions (especially, at higher energy values) there is not practically any actual interference between Coulomb and strong interactions. In any event only one of these interactions is effective according to impact parameter value and the corresponding probabilities of individual contributions to elastic differential cross section may be added. However, in such a case it is necessary to take into account possible proton dimensions in collision instant. According to contemporary knowledge protons consist evidently of other objects that must be in mutual interaction. It is also necessary to expect that the particles having special value of spin must exhibit some internal dynamics. We are to accept that the proton may exist in different spontaneously changeable internal states that may have also different shapes and dimensions (as it has been proposed earlier in [38]). And we shall attempt to test whether these dimensional characteristics may become evident in elastic collision characteristics.

To make use of the given idea in the analysis of elastic proton-proton processes let us assume that a colliding proton may exist in $n$ different states, each being characterized by maximal possible dimension $d_{k}\left(d_{1} \geq \ldots \geq d_{k} \geq \ldots \geq d_{n}\right)$. The corresponding probabilities that proton is in such a state in the moment of interaction are then $p_{k}\left(\sum_{k} p_{k}=1\right)$.

If two such protons or other similar objects collide the different pairs of states may interact; the individual probabilities of such collision channels (or collision states) being equal to

$$
r_{k, l}= \begin{cases}p_{k} p_{l} & \text { if } k=l \text { or the colliding object are not identical } \\ 2 p_{k} p_{l} & \text { if } k \neq l \text { and the colliding object are identical, } k<l\end{cases}
$$

The factor 2 in the last relation follows from the fact that for $k \neq l$ and identical colliding objects the cases with interchanged collision types $k, l$ and $l, k$ are the same and the corresponding probabilities may be summed but we need to introduce convention $k<l$ to count each distinct collision type only once.

\subsection{Nuclear and Coulomb scattering}

As to the strong interactions (taken as contact ones) the maximum effective impact parameter for which two protons may still interact hadronically in corresponding collisions will be then equal to

$$
B_{k, l}=\left(d_{k}+d_{l}\right) / 2 .
$$


The two indexes $k$ and $l$ in $r_{k, l}$ and $B_{k, l}$ may be for convenience substituted by only one index $j$ using a one-to-one correspondence $(k, l) \leftrightarrow j$. In other words, if one object in a state $k$ collide with an another one in a state $l$ then this always implies unique collision type $j$ and vice versa. It is obvious that $\sum_{j} r_{j}=1$.

It is necessary to expect that for each $j$-th collision channel at any given impact parameter $b<B_{j}$ the value of scattering angle (or equivalently $|t|$ ) of two elastically (and hadronically only) scattered protons will belong to a limited interval of values beginning always with zero value. The corresponding frequency of individual values of scattering angle will go to a maximum and will diminish again to zero; the interval being reduced to one point at $b=B_{j}$. However, in the following very preliminary (and simplified) analysis of experimental data we have substituted the given interval by the corresponding mean value. Let us denote the corresponding function as $\bar{t}_{j}(b)$ which smoothly fall from zero to some lower negative values when $b$ will change from $B_{j}$ to lower values. The inverse function will be denoted as $\bar{b}_{j}(t)$.

We may write for partial elastic hadronic cross section corresponding to a $j$-th collision state following expression

$$
\frac{d \sigma_{j}^{N}(t)}{d t}=2 \pi P_{j}^{e l}\left(\bar{b}_{j}(t)\right) \bar{b}_{j}(t) \frac{d \bar{b}_{j}(t)}{d t}
$$

where the factor $P_{j}^{e l}(b)$ represents individual probability of elastic processes at corresponding impact parameter values $b$ if cylindrical symmetry in impact parameter dependence has been assumed. The elastic hadronic differential cross section is then given by the sum of individual contributions from all the collision states $j$ :

$$
\frac{d \sigma^{N}(t)}{d t}=\sum_{j} r_{j} \frac{d \sigma_{j}^{N}(t)}{d t} .
$$

Parameters $r_{j}$ in the last relation represent the weights (probabilities) of individual collision channels.

The behavior of the given pair of two colliding protons depends then on probabilities $p_{k}$ of individual states, their dimension values $d_{k}$ and on two series of functions $P_{j}^{e l}(b)$ and $\bar{b}_{j}(t)$ (or $\bar{t}_{j}(b)$ ). Their values or shapes are to be derived from corresponding experimental data. However, the function $P_{j}^{e l}(b)$ may be expressed as the product of two functions

$$
P_{j}^{e l}(b)=P_{j}^{t o t}(b) P_{j}^{r a t}(b)
$$

where $P_{j}^{\text {tot }}(b)$ is the probability of any mutual hadronic particle interaction (elastic as well as inelastic) at impact parameter $b$ corresponding to a $j$-th collision type while $P_{j}^{r a t}(b)$ represents the corresponding ratio of elastic hadronic processes from all possible hadronic interactions. Both the functions $P_{j}^{\text {tot }}(b)$ and $P_{j}^{r a t}(b)$ are evidently monotonous. The former one is to be non-increasing function of impact parameter $b$ while the latter one is non-decreasing in dependence on $b \in\left\langle 0, B_{j}\right\rangle$. The monotony of the functions brings very important 
simplification in the choice of parametrization of both the new functions; their values moving in the whole interval $\langle 0,1\rangle$. The probability of any inelastic process may be then defined as

$$
P_{j}^{\text {inel }}(b)=P_{j}^{\text {tot }}(b)-P_{j}^{e l}(b)
$$

It must hold always $P_{j}^{\text {tot }}(b)=P_{j}^{e l}(b)=P_{j}^{\text {inel }}(b)=0$ for any $b \geq B_{j}$. This will allow us to integrate in corresponding cases over finite interval of impact parameter $\left\langle 0, B_{j}\right\rangle$ instead of infinite interval $\langle 0, \infty\rangle$. We will use this fact in the following quite frequently; it also simplifies numerical calculations of corresponding expressions.

The (integrated) elastic hadronic cross section may be written with the help of Eq. (20) and Eq. (21) as

$$
\sigma^{e l, N}=\int_{t_{\min }}^{0} d t \frac{d \sigma^{N}(t)}{d t}=\sum_{j} r_{j} \sigma_{j}^{e l, N}
$$

where we have introduced elastic hadronic cross section just for $j$-th collision channel

$$
\sigma_{j}^{e l, N}=2 \pi \int_{0}^{B_{j}} d b P_{j}^{e l}(b) b
$$

In the given model it is possible to derive also total hadronic cross sections for individual collision types $\sigma_{j}^{t o t, N}$ from elastic experimental data without adding any further assumption; it holds

$$
\sigma_{j}^{t o t, N}=2 \pi \int_{0}^{B_{j}} d b P_{j}^{t o t}(b) b .
$$

The total hadronic cross section may be then written again as a sum of contributions from all the collision channels

$$
\sigma^{t o t, N}=\sum_{j} r_{j} \sigma_{j}^{t o t, N}
$$

And using the relation (23) the corresponding values for inelastic cross section may be established, too.

The mutual elastic collisions between two protons at smaller scattering angles (smaller momentum transfers $|t|$ ) are caused, however, not only by strong interactions that may be interpreted practically as contact but also by mutual Coulomb forces acting at distance. One can express then the experimentally measured elastic differential cross section as the sum of the two given interactions

$$
\frac{d \sigma^{C+N}(t)}{d t}=\sum_{j} r_{j} \frac{d \sigma_{j}^{N}(t)}{d t}+\frac{d \sigma^{C}(t)}{d t}
$$


where the Coulomb differential cross section will be established directly by fitting experimental data. It will not be determined with the help of formfactors from the standard Coulomb potential valid for pointlike particles as such approach does not correspond to reality. The infinite Coulomb elastic differential cross section for $t=0$ may be theoretically obtained at infinite distance while in real experiments the zero value of $t$ may exist inside narrow particle beam as combined effect from different surrounding scattering centers. The similar difference may concern, of course, also the frequencies for higher values of $|t|$ due to asymmetric positions of charged partons in individual protons.

In our model we have introduced some free parameters and some unknown functions which are to be determined from corresponding measured elastic differential cross section using formula (28). It is necessary to fit maximal dimensions $d_{k}$ of all the (considered) hadron states and their corresponding probabilities $p_{k}$ at the moment of collision. We also need to parametrize and then to fit three monotonic functions $P_{j}^{t o t}(b), P_{j}^{r a t}(b)$ and $\bar{b}_{j}(t)$ (or $\bar{t}_{j}(b)$ ) for each corresponding $j$-th hadronic collision channel. It is also the Coulomb interaction effect $\frac{d \sigma^{C}(t)}{d t}$ which needs to be determined from data. Several other very interesting and physically significant quantities may be then calculated from these parameters and functions as it has been shown in preceding.

\subsection{Analysis of experimental data}

We shall apply the given probabilistic model as we have already mentioned to the data obtained at ISR at CERN at the energy of $53 \mathrm{GeV}[33,34]$ (the same data as made use of in Sec. 4). We shall try to show that two proton states exhibiting the largest dimensions may be responsible for the part of differential elastic cross section data corresponding to $|t| \in(0 ., 1.25) \mathrm{GeV}^{2}$; see the corresponding part of experimental points shown in Fig. 2. As in the lower part of $|t|$ the density of measured points has been very great only one fifth of experimental points has been pictured in the interval $(0 ., 0.45) \mathrm{GeV}^{2}$.

If one proton in $k$-state $(k=1,2)$ collides with another proton in $l$-state $(l=1,2)$ we may define collision state $j$ using a following one-to-one correspondence $(k, l) \leftrightarrow j(k<l):(1,1) \leftrightarrow 1$, $(1,2) \leftrightarrow 2$ and $(2,2) \leftrightarrow 3$. We have thus three distinct collision types that will be responsible for the given part of elastic differential cross section.

It is, of course, necessary to parametrize suitably the corresponding functions used in the description of the given process. The following parameterizations of three monotonous functions $P_{j}^{t o t}(b), P_{j}^{r a t}(b)$ and $\bar{b}_{j}(t)$ have been used for individual collision channels

$$
P_{j}^{t o t}(b)= \begin{cases}1 & \text { if } 0 \leq b \leq \mu_{0, j} \\ \frac{e^{-\left(\mu_{1, j}\left(b-\mu_{0, j}\right)\right)^{\mu_{2, j}}\left(1+\mu_{3, j}\right)}}{1+\mu_{3, j} e^{-\left(\mu_{1, j}\left(b-\mu_{0, j}\right)\right)^{\mu_{2, j}}}} & \text { if } \mu_{0, j}<b<B_{j} \\ 0 & \text { if } B_{j} \leq b\end{cases}
$$




$$
\begin{gathered}
P_{j}^{r a t}(b)=\frac{e^{-\left(v_{0, j}\left(B_{j}-b\right)\right)^{v_{1, j}}}\left(1+v_{2, j}\right)}{1+v_{2, j} e^{-\left(v_{0, j}\left(B_{j}-b\right)\right)^{v_{1, j}}}} \\
\bar{b}_{j}(t)=B_{j}\left(\frac{2}{\pi} \arccos \left[\left(\frac{|t|}{\eta_{0, j}}\right)^{1 / \eta_{2, j}}\right]\right)^{1 / \eta_{1, j}}
\end{gathered}
$$

where $\mu_{0, j}, \mu_{1, j}, \mu_{2, j}, \mu_{3, j} ; v_{0, j}, v_{1, j}, v_{2, j} ; \eta_{0, j}, \eta_{1, j}, \eta_{2, j} \quad(j=1,2,3)$ are free parameters that are to be determined from experimental data together with parameters $p_{k}$ and $d_{k}(k=1,2)$.

As it has been already mentioned also the Coulomb interaction effect is to be determined from corresponding experimental data. To enable precise fit the following parametrization has been chosen

$$
\frac{d \sigma^{C}(t)}{d t}=\xi_{0}\left(1+\left(\xi_{1}|t|\right)^{\xi_{2}}\right) e^{-\xi_{3}|t|}+\xi_{4} e^{-\left(\xi_{5}|t|\right)^{\xi_{6}}} \frac{1+\xi_{7}}{1+\xi_{7} e^{-\left(\xi_{5}|t|\right)^{\xi_{6}}}}
$$

where $\xi_{i}(i=0, . ., 7)$ are additional free parameters that are to be derived from corresponding experimental data.

The number of free parameters may seem to be rather high. However, this is quite irrelevant at this stage of our research when quite new physical ideas are looked for and tested. The goal of our effort consists in principle in describing the characteristics of partial structures to a sufficient detail, to initiate further analysis with the help of other experiments.

The results of the corresponding fit are shown in Fig. 2. The probabilistic model can be fitted to experimental data practically in the whole considered interval of $|t| \in(0,1.25) \mathrm{GeV}^{2}$. Hadronic differential cross sections $\frac{d \sigma_{j}^{N}(t)}{d t}$ given by Eq. (20) for all the three collision types are plotted in Fig. 2, too. Only their parts given by $r_{j}$ parameters contribute to complete differential cross section $\frac{d \sigma^{C+N}(t)}{d t}$. The Coulomb differential cross section $\frac{d \sigma^{C}(t)}{d t}$ given by parametrization (32) is also shown in Fig. 2.

The following values of individual free parameters have been obtained on the basis of our analysis. The frequencies of two internal states considered in our fit to experimental data having the greatest dimensions are given by

$$
p_{1}=0.48, \quad p_{2}=0.39
$$

and the corresponding dimensions are

$$
d_{1}=2.50 \mathrm{fm}, \quad d_{2}=2.29 \mathrm{fm}
$$



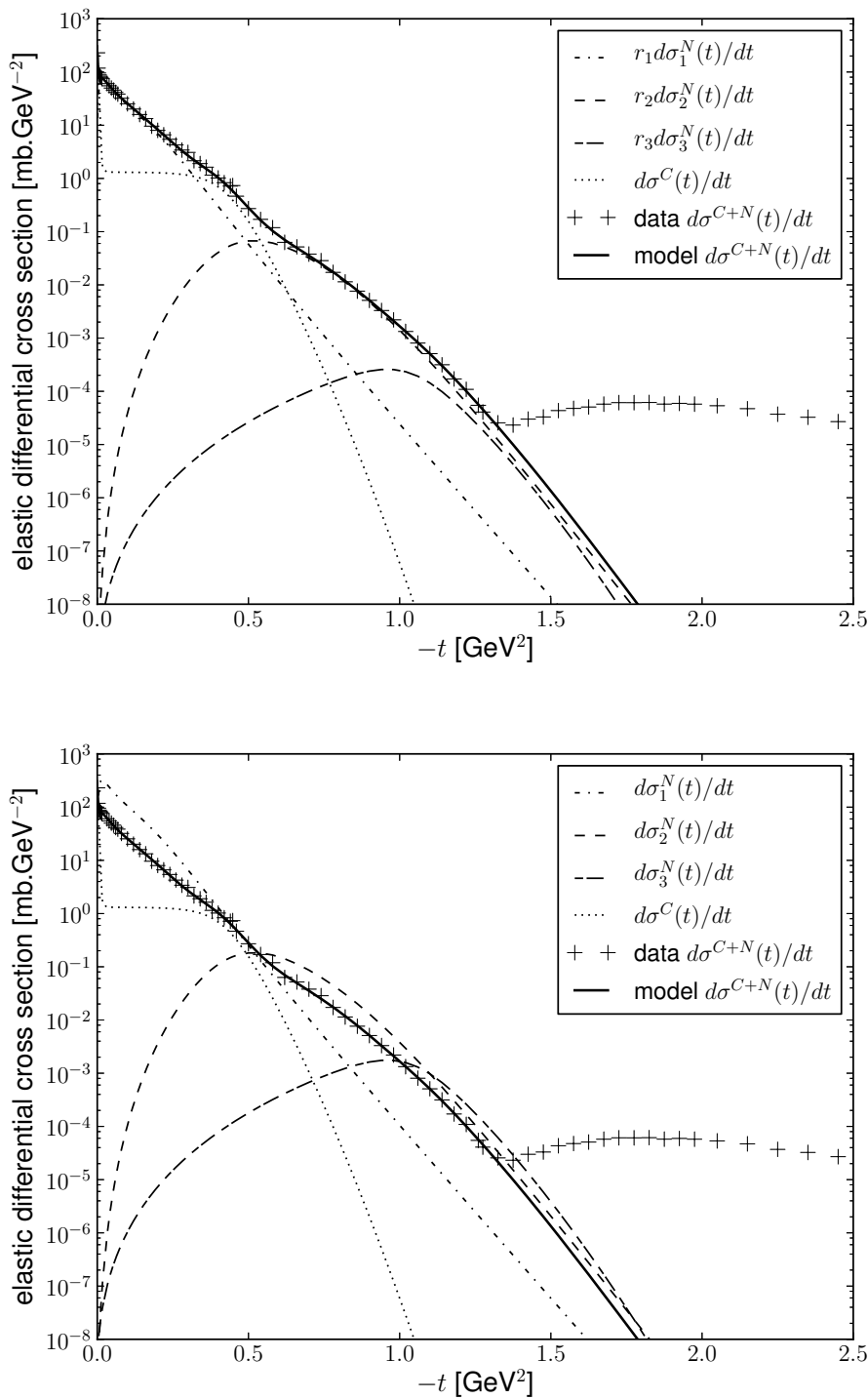

Figure 2. Differential elastic cross sections for proton-proton scattering at energy of $53 \mathrm{GeV}$; individual points - experimental data, full line - our probabilistic model fitted to experimental data, dashed line - Coulomb differential cross section $d \sigma^{C}(t) / d t$, three other lines - individual hadronic collision types (top - contributions $r_{j} d \sigma_{j}^{N}(t) / d t$ to complete differential cross $d \sigma^{C+N}(t) / d t$, bottom - individual differential cross sections $\left.d \sigma_{j}^{N}(t) / d t\right)$.

The functions $P_{j}^{\text {tot }}(b)$ and $P_{j}^{r a t}(b)$ (see Eqs. (29) and (30)) representing the $b$-dependences of probabilities of total and elastic collisions together with function $\bar{t}_{j}(b)$ (inverse function of 
$\bar{b}_{j}(t)$ given by Eq. (31)) are shown in Fig. 3. The values of free parameters in the functions describing the elastic scattering caused by strong interaction are shown in Table 3 for all the three considered collision states.

\begin{tabular}{cllccc}
\hline & & \multicolumn{1}{c}{$\mathrm{j}$} & $\mathbf{1}$ & $\mathbf{2}$ & $\mathbf{3}$ \\
\hline \multirow{4}{*}{$P_{j}^{\text {tot }}(b)$} & $\mu_{0, j}$ & {$[\mathrm{fm}]$} & 0.70 & 0.59 & 0.50 \\
& $\mu_{1, j}$ & {$\left[\mathrm{fm}^{-1}\right]$} & 0.95 & 2.35 & 4.34 \\
& $\mu_{2, j}$ & {$[1]$} & 5.90 & 2.55 & 1.69 \\
& $\mu_{3, j}$ & {$[1]$} & 193. & 995. & 15. \\
\hline \multirow{3}{*}{$P_{j}^{\text {rat }}(b)$} & $v_{0, j}$ & {$\left[\mathrm{fm}^{-1}\right]$} & 2.03 & 2.40 & 1.80 \\
& $v_{1, j}$ & {$[1]$} & 4.34 & 3.38 & 5.65 \\
& $v_{2, j}$ & {$[1]$} & 20.7 & 144. & 3742. \\
\hline \multirow{3}{*}{$\bar{b}_{j}(t)$} & $\eta_{0, j}$ & {$\left[\mathrm{GeV}^{2}\right]$} & 24.8 & 26.8 & 35.3 \\
& $\eta_{1, j}$ & {$[1]$} & 0.97 & 0.44 & 0.47 \\
& $\eta_{2, j}$ & {$[1]$} & 5.15 & 2.63 & 2.85 \\
\hline
\end{tabular}

Table 3. Values of free parameters of monotonous functions $P_{j}^{\text {tot }}(b), P_{j}^{r a t}(b)$ and $\bar{b}_{j}(t)$ for all the three collision types given by parametrizations (29), (30) and (31).

The additional effect caused by electric charges is then characterized by function (32) that contains some further free parameters. Their values obtained by fitting the given experimental data are

$$
\begin{gathered}
\xi_{0}=491 \mathrm{mb} \cdot \mathrm{GeV}^{-2}, \xi_{1}=265 \mathrm{GeV}^{-2}, \xi_{2}=3.70, \xi_{3}=742 \mathrm{GeV}^{-2}, \\
\xi_{4}=1.32 \mathrm{mb} \cdot \mathrm{GeV}^{-2}, \quad \xi_{5}=6.14 \mathrm{GeV}^{-2}, \xi_{6}=1.70, \xi_{7}=106 .
\end{gathered}
$$

Once we have determined all the free parameters and unknown functions from the fit we may calculate several other physically significant quantities. Let us start with quantities which we can calculate for all the three individual collision states considered in our fit.

The parameters $r_{j}$ (see Eq. (18)) determining the contribution of individual channels (their probabilities) are in Table 4 . Corresponding maximal effective impact parameters $B_{j}$ for which protons might still interact via hadronic interaction calculated from Eq. (19) are shown, too; they are around $2.4 \mathrm{fm}$ slightly different for each collision type $j$. Further total, elastic and inelastic hadronic cross sections having been calculated for all three collision states $j$ are introduced in Table 4.

According to our very very rough model a colliding proton may be in one of two considered internal states with probability $p_{1}+p_{2}=0.86$. It means that in $14 \%$ of cases the proton is to be in different internal states with different maximal dimensions. The proton collisions go then in the given three channels with the probability $\sum_{j=1}^{3} r_{j}=p_{1}^{2}+2 p_{1} p_{2}+p_{2}^{2}=0.75$. The additional internal states may be responsible for measured differential cross section outside our considered $t$-range (for $|t|>1.25 \mathrm{GeV}^{2}$ ); partially also in combination with two already considered states, which is in agreement with preliminary tests done already by us, too. It means that the actual total and inelastic cross sections will be higher than the values 

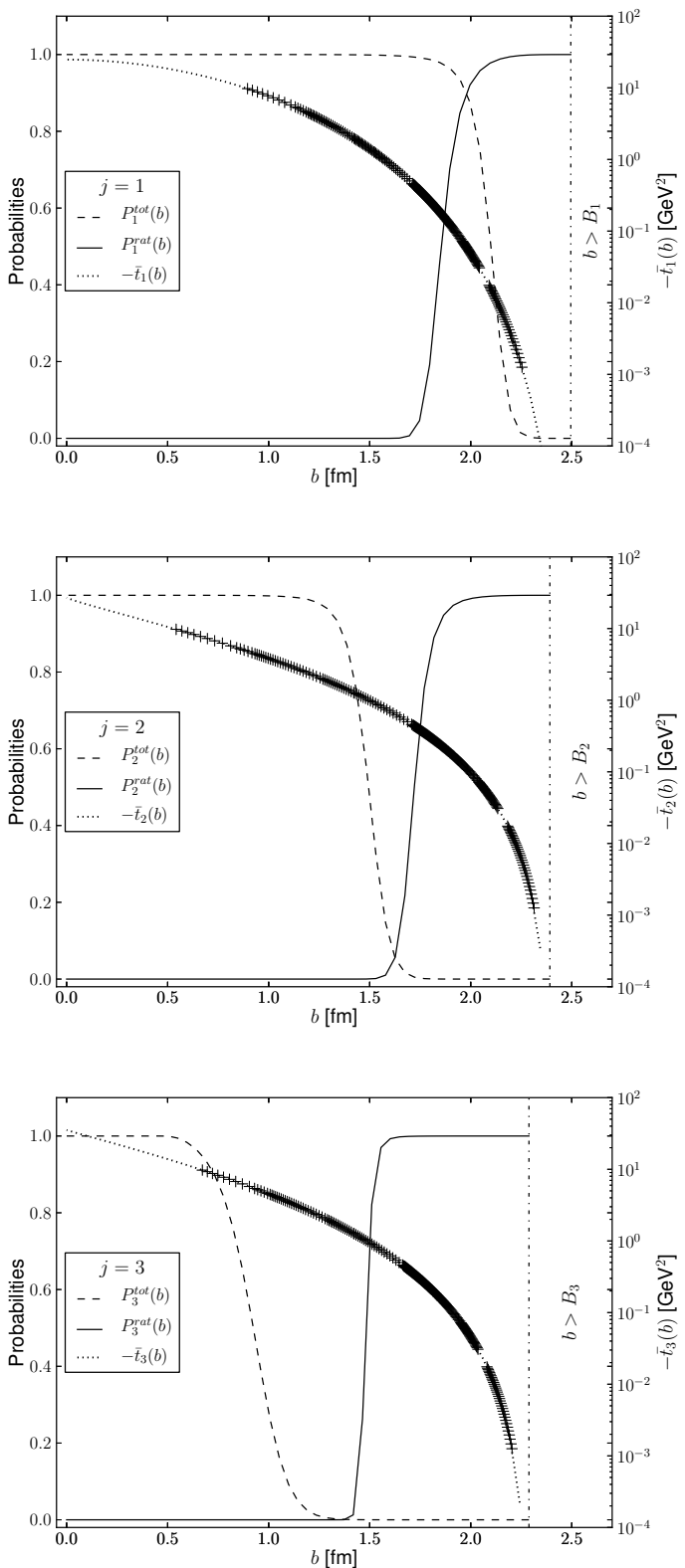

Figure 3. Functions $P_{j}^{\text {tot }}(b)$ and $P_{j}^{\text {rat }}(b)$ with opposite monotony (dashed and full lines) and functions $\left|\bar{t}_{j}(b)\right|$ (dotted line) for individual collision types (top $j=1$, middle $j=2$, bottom $j=3$ ). Individual points lying on $\left|\bar{t}_{j}(b)\right|$ curves correspond to values of impact parameters $b$ at experimentally established values of $t$ calculated using functions $\bar{b}_{j}(t)$. 


\begin{tabular}{clcccc}
\hline $\mathrm{j}$ & & $\mathbf{1}$ & $\mathbf{2}$ & $\mathbf{3}$ & $\sum_{j=1}^{3}$ \\
$\mathrm{k}, 1$ & & $\mathbf{1 , 1}$ & $\mathbf{1 , 2}$ & $\mathbf{2 , 2}$ & 0.75 \\
\hline$r_{j}$ & {$[1]$} & 0.23 & 0.37 & 0.15 & 0.79 \\
$B_{j}$ & {$[\mathrm{fm}]$} & 2.50 & 2.39 & 2.29 & - \\
\hline$\sigma_{j}^{\text {tot, } N}$ & {$[\mathrm{mb}]$} & 137. & 69.7 & 27.1 & - \\
$\sigma_{j}^{\text {el,N }}$ & {$[\mathrm{mb}]$} & 28.1 & $6.2 \times 10^{-2}$ & $7.2 \times 10^{-4}$ & - \\
$\sigma_{j}^{\text {inel }}$ & {$[\mathrm{mb}]$} & 109. & 69.7 & 27.1 & - \\
\hline$r_{j} \sigma_{j}^{\text {tot,N }}$ & {$[\mathrm{mb}]$} & 31.5 & 25.7 & 4.0 & 61.2 \\
$r_{j} \sigma_{j}^{e l, N}$ & {$[\mathrm{mb}]$} & 6.5 & $2.3 \times 10^{-2}$ & $1.1 \times 10^{-4}$ & 6.5 \\
$r_{j} \sigma_{j}^{\text {inel }}$ & {$[\mathrm{mb}]$} & 25.0 & 25.7 & 4.0 & 54.7 \\
\hline
\end{tabular}

Table 4. Values of some physically significant quantities obtained as a result of the probabilistic ontological model fitted to corresponding experimental data at energy of $53 \mathrm{GeV}$.

introduced in Table 4: $\sigma^{\text {tot, } N}>\sum_{j=1}^{3} r_{j} \sigma_{j}^{t o t, N}=61.2 \mathrm{mb} \quad$ (resp. $\sigma^{i n e l, N}>\sum_{j=1}^{3} r_{j} \sigma_{j}^{i n e l, N}=$ $54.7 \mathrm{mb}$ ), while the increase of elastic cross section may be neglected. The given values may be, of course, influenced by the very rough approximation neglecting the distribution of momentum transverses at any impact parameter value.

It is evident that according to our probabilistic model the Coulomb effect appears as significant till $|t| \cong 0.5 \mathrm{GeV}^{2}$, which might throw quite new light to the structure of charge distribution in individual protons and contribute fundamentally to our understanding of the internal structure of hadron objects.

The presented model has been based, of course, on one very simplifying and rough assumption $\left(\bar{t}_{j}(b)\right.$ - relating always only one momentum value to each impact parameter), which might significantly influence the corresponding numerical results. We are working already on the model that will respect the existence of momentum transfer spectrum for any impact parameter value, which should allow much more realistic fit of experimental data on the given basis. Also the fitting of experimental data at other energy values will be performed; the fits for higher values of $|t|$ will be done, too.

\section{Contemporary quantum physics and metaphysics}

We have mentioned already in the preceding that the new results require for the physicists to return to ontological basis the classical physics was based on. This basic approach changed fundamentally in the beginning of modern period when Descartes formulated his mathematically-philosophical concept in which any linkage between human knowledge and ontological approach to matter world was practically excluded. Any knowledge of matter existence started to be based fully on human thinking. Also the participation of human senses was excluded at that time, which was criticized by some philosophers. It led then to the positivism that influenced fully the thinking of European society in the course of the 19th century.

In the middle of the 19th century the given thinking dominated also scientific approaches and scientific knowledge. Boltzmann started it when he denoted one phenomenological characteristics of the system consisting of a great number of particles as natural law. It is possible to say that the given way was accomplished when Bohr formulated his Copenhagen quantum mechanics in 1927. He started from Schrödinger equation proposed in 1925 which 
itself was fully acceptable as the same results followed from it as from the classical concept of Galileo and Newton (only the set of corresponding states was smaller); see short paragraph (with corresponding quotations) in Sec. 1. However, Bohr deformed significantly physical conclusions (following from proper Schröedinger equation) by adding further very strong assumptions without any actual reason, which influenced fundamentally the evolution of quantum physics in the 20th century.

The corresponding (quantum) era of physical research started in principle at the break of the 19th and 20th centuries when black-body radiation was being intensively studied and at the same time new phenomena at the level of microscopic world were discovered ( $X$ and gamma radiations and electron (1895-1897)); and further, quantum energy transfer (1900) and photon existence (1904) were predicted. A broad space was opened for formulation of new hypotheses that could not be tested directly but only on the basis of indirect effects established with the help of macroscopic measuring devices. In such a situation the phenomenological models seemed to be very advantageous.

However, to understand the possibility of scientific knowledge it is necessary to realize what are the approaches of scientific research. As to the region of physical research it is possible to say that it is based on falsification approach. The basic step consists then in formulating some more general statements or unifying hypotheses with the help of our reason on the basis of observation and measurement; with the help of the approach making use of logical induction, or intuition. The goal of further approaches and analyses consists then in deriving all possible consequences that follow from a given hypothesis (or a set of hypotheses). As such hypotheses start always from a limited set of our pieces of knowledge it is clear in some cases immediately that they cannot sustain in further considerations. Generally, it is necessary to derive for any hypothesis all possible consequences with the help of logical deduction and to analyze, whether some logical contradictions between individual consequences do not exist, and further to compare these consequences to all possible observations of natural phenomena. If a contradiction is found the given hypothesis (or a set of hypotheses) must be refused, resp. modified, so as the given contradiction might be removed. If one does not come after sufficient falsifying effort to any contradiction the given set of hypotheses may be denoted as plausible; and it is possible to start from it in further considerations about the natural laws concerning the world and human being.

It follows from the preceding that the falsification approach represents important and practically basic knowledge method based on human reason, as K. Popper (1902-94) presented in the first half of the 20th century. One is never justified to denote our reason knowledge for a verified truth, as one can never grant that in following steps a logical contradiction or a contradiction to nature observation will not appear. On the other side one must accept any non-falsified hypothesis as plausible, even if it is in contradiction to another plausible hypothesis. All these statements or hypotheses must be fully tolerated. The decision concerning the preference of one of them must be left to other logical and experimental analyses. On the other side in contradiction to contemporary requirements of some human groups any falsified statement cannot be tolerated and must be decisively refused.

The preceding arguments have thrown new light also to the principle of falsifiability propagated in the region of physical research in the last century. The requirement of falsification tests to any statement has been interpreted as the possibility to prove the validity 
of the given statement, which contradicts the possibilities of our reason knowledge. At least some positive test should be necessary to admit the given statement as plausible; however, it can never represent any proof of its validity.

In the 20th century purely phenomenological view was taken as the knowledge basis and any ontological aspects have not been respected. Evidently mutually contradicting assumptions were being applied especially to elastic collision processes. It follows from these facts that the corresponding metaphysical view must be based on ontological view respecting fully our experience with matter nature.

\section{Some open questions}

One of the most important pieces of knowledge introduced in preceding sections is the fact that there is fundamental difference between Coulomb forces and strong (nuclear) interaction; the former acting at a distance and the latter representing a contact force. To interpret this force as the effect of some potential may be misleading. And it is the task of contemporary research to find some new approaches how to describe successfully the given phenomenon. The analyses of elastic collisions between the particles exhibiting corresponding interaction may be very helpful in this direction. It is even possible to say that the further progress of future quantum physics is strongly bound to the problem of particle structure. The existence and emergence of quantum states where not only the values of energy but also the values of angular momentum are quantized may be hardly understood without the progress in this region.

It follows from the preceding that it will be probably necessary to distinguish between two kinds of quantum states: one relating to the quantum states of atoms and the other relating to existence of individual matter objects characterized by special values of rest energy and angular momentum. The former relates to the mutual properties (interaction) of electrons and protons and the latter to mutual interactions of strongly interacting objects. It has been shown that the Coulomb interaction itself cannot explain the emergence of quantum states and that some additional interaction must exist between protons (nucleons) and electrons. And one should ask whether a kind of weak contact forces does not exist in addition to strong (nuclear) contact ones.

The existence of contact forces has opened a new question: How to describe the given situation with the help of corresponding mathematical models. It is evident that it will be necessary to limit the solutions of Schrödinger equation to subsystems exhibiting continuous evolution. This approach should be used in individual subsystems where no sudden changes (caused by contact forces) are occurring; and to describe the effect of contact forces probably as the passage to another subsystem being described again as developing continuously. The given situation may be represented in the Hilbert space consisting of individual mutually orthogonal subspaces defined with the help of corresponding Schrödinger equation describing respective continuous evolution.

The representation of physical processes in a suitable Hilbert space is surely very helpful. It means, of course, that we must represent the states corresponding to quantities conserving during the whole time evolution in mutually orthogonal subspaces. It concerns mainly two quantities: energy and angular momentum. The corresponding trajectories should always 
belong to mutually orthogonal subspaces. It may concern also the representation of states characterized by impact parameter values in the case of collision processes.

In any case it is possible to say that we have pass to the other ontological level that differs from that the classical physics has been based on and opens quite new questions. It is also the fact that these basic particles are to be characterized by special values of spins, testifying that these particles exhibit special internal dynamics which may lead to the existence of their different internal states. It has been demonstrated that these characteristics may be studied with the help of corresponding elastic collisions processes.

The given results might then help in revealing the internal dynamics running in corresponding objects. It is evident that practically all particles existing in the nature must be taken as complex quantum objects. The new results may contribute in looking for the characteristics of constituents of which the given particles consist and which are responsible for their structures. At the present the main attention is to be devoted to the protons and other hadrons; especially the reasons for their stability or instability should be analyzed.

From the presented results it is also possible to conclude that the contemporary quark theory having been proposed on the basis of phenomenological description of corresponding physical situation may hardly represent suitable basis to the given goal. It is, of course, necessary to expect that some basic objects (some "quasi-quarks") should exist that should exhibit some very strong (superstrong) contact interaction. This interaction kind might be responsible for forming some conglomerates equivalent to the so called partons corresponding in principle to experimentally observable hadrons. These partons might form changeable structures (being held mutually together by standard strong forces) of individual hadrons while they might be kicked out from the given object when sufficient amount of kinetic energy has been furnished to them in the corresponding collision event.

The preceding considerations have been based on the ontological approach respecting the basic matter properties when it has been shown that the past refusal of ontology led to key mistakes in the physics of the 20th century. One must be very careful in formulating and testing different hypotheses when some quite new properties of nanoscopic objects should be taken into account (e.g., the existence of contact forces). However, the ontological basis (including causal sequentiality) in the description of matter world should not be abandoned.

\section{Conclusion}

Even if the Schrödinger equation might represent in principle basic theory of the whole matter reality it corresponds to approximate phenomenological description only. Especially, it is not possible to explain at all how a quantum state may emerge. E.g., it is evident that the hydrogen atom arises always when slowly moving electron and proton meet and are attracted mutually by Coulomb force, which cannot be theoretically reasoned. It may occurred only if a kind of repulsive force between two given objects or a kind of impenetrability of proton having certain dimensions is to be involved. It means that the structure of protons (and other hadrons) represents indivisible part of the contemporary quantum physics.

Consequently, the concept of the hydrogen atom formed by proton and electron orbiting around should be taken hardly as acceptable. The adhesive merging of both the constituents must be regarded as much more probable concept. Here, of course, the existence of different internal proton structures should be responsible for divers quantum states of hydrogen 
atoms. It is necessary to look for the experiments how to get corresponding pieces of knowledge.

It has been demonstrated that some characteristics of these different proton structures might be derived from the data of elastic two-proton collisions. However, the earlier antiontological proton properties have been obtained on the basis of phenomenological models where some arbitrary additional assumptions have been added. The standard ontological characteristics have been obtained when the eikonal model enabling to test the dependence on impact parameter value has been made use of. When this ontological model has been further generalized (and corresponding probabilities have been derived directly from measured values) it has been shown that the momentum transfer dependence of differential cross section may be reconstructed as the consequence of colliding protons exhibiting a series of structures of different external dimensions. It opens new way how the existence of quantum atom states might be interpreted on much more realistic basis.

It represents also important argument that the purely phenomenological approach to physical reality should be abandoned as quite insufficient. It is necessary to return to ontological approach on which all successes of the classical physics were based and from which practically all world civilization emerged. Some interesting orientation results have been already obtained with the help of the mentioned very rough model. Now the attention is to be devoted to its generalization to correspond fully to all ontological requirements and not to involve any unphysical limitation.

The given results should contribute mainly to understand better the existence of atom quantum states; and how they may be influenced by proton structure. It is necessary to analyze corresponding data from experiments that might help in this direction; mainly elastic collision experiments may be very helpful. However, it is not more possible to look for a phenomenological description of measured values only, but for the interpretation of corresponding processes on ontological basis.

However, it may be also helpful to answer the question how it was possible that the Copenhagen alternative was influencing scientific thinking in greater part of the past century. It followed from the fact that two different kinds of quantum physics have existed; one based on the Copenhagen quantum mechanics and looking for the support of quantum paradoxes and the other one solving in principle successfully different physical and technological problems on the basis of standard Schrödinger approach (no additional assumption having been added - without mentioning it explicitly). It is possible to say that it followed from the fact that the scientific thinking in the modern period was fundamentally influenced by mathematical philosophy of Descartes refusing ontological approach. It was also the reason why also Einstein's criticism based on ontological argument has been refused by scientific community. Our main contemporary task consists in devoting more attention to the ontological properties of physical objects.

\section{Author details}

Miloš V. Lokajiččk*, Vojtěch Kundrát and Jiří Procházka

* Address all correspondence to: lokaj@fzu.cz; kundrat@fzu.cz; prochazka@fzu.cz Institute of Physics of the AS CR, Prague, Czech Republic 


\section{References}

[1] E. Schrödinger: Quantisierung als Eigenwertproblem; Ann. Phys. 79, 361-76; 489-527; 80, 437-90; 81, 109-39 (1926).

[2] N. Bohr: The quantum postulate and the development of atomic theory; Nature 121, 580-90 (1928).

[3] W. Pauli: Die allgemeinen Prinzipien der Wellenmechanik; Handbuch der Physik XXIV, Springer, Berlin, p. 140 (1933).

[4] A. Einstein, B. Podolsky, N. Rosen: Can quantum-mechanical description of physical reality be considered complete?; Phys. Rev. 47, 777-80 (1935).

[5] N. Bohr: Can quantum-mechanical description of physical reality be considered complete?; Phys. Rev. 48, 696-702 (1935).

[6] J. von Neumann: Mathematische Grundlagen der Quantenmechanik; Springer (1932).

[7] Grete Herrmann: Die Naturphilosophischen Grundlagen der Quantenmechanik; Abhandlungen der Fries'schen Schule 6, 75-152 (1935).

[8] D. Bohm: A suggested interpretation of the quantum theory in terms of "hidden variables"; Phys. Rev. 85, 180-93 (1952).

[9] J. S. Bell: On the Einstein Podolsky Rosen paradox; Physics 1, 195-200 (1964).

[10] A. Aspect, P. Grangier, G. Roger: Experimental realization of Einstein-Podolsky-Rosen-Bohm Gedankenexperiment: A new violation of Bell's inequalities; Phys. Rev. Lett. 49, 91-4 (1982).

[11] M. V. Lokajíček: Locality problem, Bell's inequalities and EPR experiments; /arXiv:quant-ph/9808005 (1998).

[12] E. E. Rosinger: George Boole and the Bell inequalities; /arXiv:quant-ph/0406004.

[13] G. Boole: On the Theory of Probabilities; Philos. Trans.; R. Soc., London 152, 225-52 (1862).

[14] N. Vorob'ev: Theor. Probab. Appl. 7, 147 (1952).

[15] M. V. Lokajíček: The assumption in Bell's inequalities and entanglement problem; J. Comp. Theor. Nanosci. (accepted for publication), /arXiv:1108.0922.

[16] M. V. Lokajíček: Einstein-Bohr controversy and theory of hidden variables; NeuroQuantology (section: Basics of Quantum Physics) 8 (2010), issue 4, 638-45; see also /arXiv:1004.3005[quant-ph].

[17] M. V. Lokajíček: Einstein-Bohr controversy after 75 years, its actual solution and consequences; Some Applications of Quantum Mechanics (ed. M. R. Pahlavani), InTech Publisher (February 2012), 409-24. 
[18] U. Hoyer: Synthetische Quantentheorie; Georg Olms Verlag, Hildesheim (2002).

[19] H. Ioannidou: A new derivation of Schrödinger equation; Lett. al Nuovo Cim. 34, 453-8 (1982).

[20] M. V. Lokajíček: Schrödinger equation, classical physics and Copenhagen quantum mechanics; New Advances in Physics 1, No. 1, 69-77 (2007); see also /arxiv/quant-ph/0611176.

[21] P. Kundrát, M. Lokajíček: Three-dimensional harmonic oscillator and time evolution in quantum mechanics; Phys. Rev. A 67, art. 012104 (2003).

[22] P. Kundrát, M. Lokajíček: Irreversible time flow and Hilbert space structure; New Research in Quantum Physics (eds. Vl. Krasnoholovets, F. Columbus), Nova Science Publishers, Inc., pp. 17-41 (2004).

[23] P.D.Lax, R.S.Phillips: Scattering theory; Academic Press (1967).

[24] P.D.Lax, R.S.Phillips: Scattering theory for automorphic functions; Princeton (1976).

[25] N. Bohr: On the constitution of atoms and molecules; Philosophical Magazine 26, 1-24 (1913).

[26] V. Kundrát, M. V. Lokajíček: High-energy elastic scattering amplitude of unpolarized and charged hadrons; Z. Phys. C 63, 619-29 (1994).

[27] G. B. West and D. R. Yennie: Coulomb Interference in High-Energy Scattering; Phys. Rev. 172, 1413 (1968).

[28] J. Kašpar et al.: Phenomenological models of elastic nucleon scattering and predictions for LHC; Nucl. Phys. B 843, 84 (2011).

[29] V. Kundrát, M. Lokajíček and D. Krupa: Impact parameter structure derived from elastic collisions; Physics Letters B 544, 132 (2002).

[30] G. Giacomelli, M. Jacob: Physics at the CERN-ISR; Phys. Rep. 55, 1 (1979).

[31] V. Kundrát, M. Lokajíček Jr., M. V. Lokajíček: Are elastic collisions central or peripheral?; Czech. J. Phys. B 31, 1334 (1981).

[32] J. L. Bailly et al. (EHS-RCBC Collaboration): An impact parameter analysis of proton-proton elastic and inelastic interactions at $360 \mathrm{GeV} / \mathrm{c}$; Z. Phys. C 37, 7 (1987).

[33] J. Bystricky et al., in Nucleon-nucleon and kaon-nucleon scattering edited by H. Schopper(Landolt-Börnstein Series, Vol. 1), (Springer, Berlin, 1980).

[34] M. K. Carter, P. D. B. Collins and M. R. Whalley, Compilation of Nucleon-Nucleon and Nucleon-Antinucleon Elastic Scattering Data, RAL-86-002, preprint.

[35] R. G. Newton: Optical theorem and beyond; Am. J. Phys. 44, 639-42 (1976). 
[36] V. Barone, E. Predazzi: High-energy particle diffraction; Springer-Verlag (2002).

[37] M. V. Lokajíček, V. Kundrát: Optical theorem and elastic nucleon scattering; /arXiv:0906.3961 (see also Proc. of 13th Int. Conf., Blois Workshop; /arXiv:1002.3527 [hep-ph]).

[38] M. V. Lokajíček, V. Kundrát: Elastic pp scattering and the internal structure of colliding protons; (2009) /arXiv:0909.3199[hep-ph]. 